\title{
Thermal entanglement in three-qubit Heisenberg models
}

\author{
Xiaoguang Wang ${ }^{1,2}$ Hongchen $\mathrm{Fu}^{3}$ Allan I Solomon ${ }^{3}$ \\ 1. Institute of Physics and Astronomy, University of Aarhus, DK-8000, Aarhus C, Denmark. \\ 2. Institute for Scientific Interchange (ISI) Foundation, Viale Settimio Severo 65, I-10133 Torino, Italy \\ 3. Quantum Processes Group, The Open University, Milton Keynes, MK7 6AA, United Kingdom.
}

(October 5, 2018)

\begin{abstract}
We study pairwise thermal entanglement in three-qubit Heisenberg models and obtain analytic expressions for the concurrence. We find that thermal entanglement is absent from both the antiferromagnetic $X X Z$ model, and the ferromagnetic $X X Z$ model with anisotropy parameter $\Delta \geq 1$. Conditions for the existence of thermal entanglement are discussed in detail, as is the role of degeneracy and the effects of magnetic fields on thermal entanglement and the quantum phase transition. Specifically, we find that the magnetic field can induce entanglement in the antiferromagnetic $X X X$ model, but cannot induce entanglement in the ferromagnetic $X X X$ model.
\end{abstract}

PACS numbers: 03.65.Ud, 03.67.Lx, 75.10.Jm.

\section{INTRODUCTION}

Over the past few years much effort has been put into studying the entanglement of multipartite systems both qualitatively and quantitatively. Entangled states constitute a valuable resource in quantum information processing [1]. Quite recently, entanglement in quantum operations [2 4 and entanglement in indistinguishable fermionic and bosonic systems [5] have been considered. Entanglement in two-qubit states has been well studied in the literature, as have various kinds of threequbit entangled states 8 10]. The three-qubit entangled states have been shown to possess advantages over the two-qubit states in quantum teleportation [11], dense coding [12] and quantum cloning [13].

An interesting and natural type of entanglement, thermal entanglement, was introduced and analysed within the Heisenberg $X X X$ [14, $X X$ [15, and $X X Z$ [16] models as well as the Ising model in a magnetic field [17]. The state of the system at thermal equilibrium is represented by the density operator $\rho(T)=\exp \left(-\frac{H}{k T}\right) / Z$, where $Z=\operatorname{tr}\left[\exp \left(-\frac{H}{k T}\right)\right]$ is the partition function, $H$ the system Hamiltonian, $k$ is Boltzmann's constant which we henceforth take equal to 1 , and $T$ the temperature. As $\rho(T)$ represents a thermal state, the entanglement in the state is called thermal entanglement [14]. A complication in the analysis is that, although standard statistical physics is characterized by the partition function, determined by the eigenvalues of the system, thermal entanglement properties require in addition knowledge of the eigenstates.

The Heisenberg model has been used to simulate a quantum computer [18], as well as quantum dots [18], nuclear spins 19, electronic spins [20] and optical lattices [21]. By suitable coding, the Heisenberg interaction alone can be used for quantum computation [22]. The entanglement in the ground state of the Heisenberg model has been discussed by O'Connor and Wootters [23].

In previous studies of thermal entanglement analytical results were only available for two-qubit quantum spin models. In this paper we analyze the three-qubit case, i.e. we consider pairwise thermal entanglement in threequbit Heisenberg models.

A general 3-qubit Heisenberg $X Y Z$ model in a nonuniform magnetic field $B$ is given by:

$$
\begin{aligned}
H & =H_{X Y Z}+H_{\mathrm{mag}} \\
H_{X Y Z} & =\sum_{n=1}^{3}\left(\frac{J_{1}}{2} \sigma_{n}^{x} \sigma_{n+1}^{x}+\frac{J_{2}}{2} \sigma_{n}^{y} \sigma_{n+1}^{y}+\frac{J_{3}}{2} \sigma_{n}^{z} \sigma_{n+1}^{z}\right) \\
H_{\mathrm{mag}} & =\sum_{n=1}^{3} B_{n} \sigma_{n}^{z}
\end{aligned}
$$

We use the standard notation, detailed later, and assume a periodic boundary, identifying the subscript 4 with 1 in the above expressions. For the 3-qubit case even this most general scenario is susceptible to numerical analysis. However, in this paper we shall restrict ourselves to special cases of Eq.(11) for which we are able to provide a succinct analytic treatment.

The 3-site Heisenberg models we will study in this paper are the following:

1. The $X X$ model, corresponding to $J_{1}=J_{2}, \quad J_{3}=0$ and $\mathbf{B}=0$.

2. The $X X Z$ model, for which $J_{1}=J_{2}, J_{3} \neq 0$ and $\mathbf{B}=0$.

3. The $X X Z$ model with uniform magnetic field $\left(B_{1}=B_{2}=B_{3}\right)$.

We start in Sec. II by examining the three-qubit $X X$ model. In Sec. III, IV, and V, we study thermal entanglement in the $X X$ model, the $X X Z$ model and the $X X Z$ model in a magnetic field, respectively.

During the course of the analysis it will become clear that degeneracy plays an important role in thermal entanglement, as does the presence of magnetic fields. We find the critical temperatures involved in the quantum phase transition associated with the existence of entanglement in these quantum spin models. 


\section{THREE-QUBIT $X X$ MODEL AND ITS SOLUTION}

The three-qubit $X X$ model is described by the Hamiltonian 24

$$
\begin{aligned}
H_{X X} & =\frac{J}{2} \sum_{n=1}^{3}\left(\sigma_{n}^{x} \sigma_{n+1}^{x}+\sigma_{n}^{y} \sigma_{n+1}^{y}\right) \\
& =J \sum_{n=1}^{3}\left(\sigma_{n}^{+} \sigma_{n+1}^{-}+\sigma_{n}^{-} \sigma_{n+1}^{+}\right),
\end{aligned}
$$

where $\sigma_{n}^{\alpha}(\alpha=x, y, z)$ are the Pauli matrices of the $n$-th qubit, $\sigma_{n}^{ \pm}=\frac{1}{2}\left(\sigma_{n}^{x} \pm i \sigma_{n}^{y}\right)$ the raising and lowering operators, and $J$ is the exchange interaction constant. Positive (negative) $J$ corresponds to the antiferromagnetic (ferromagnetic) case. As signalled above, we adopt periodic boundary conditions; $\sigma_{4}^{x}=\sigma_{1}^{x}, \sigma_{4}^{y}=\sigma_{1}^{y}$. We are therefore considering a three-qubit Heisenberg ring. The $X X$ model was intensively investigated in 1960 by Lieb, Schultz, and Mattis [24]. More recently the $X X$ model has been realized in the quantum-Hall system [25], the cavity QED system [26] and quantum dot spins [27] for a quantum computer.

In order to study thermal entanglement, the first step is to obtain all the eigenvalues and eigenstates of the Hamiltonian Eq.(2). The eigenvalues themselves do not suffice to calculate the entanglement. The eigenvalue problem of the $X X$ model can be exactly solved by the Jordan-Wigner transformation [28]. In the three-qubit case the eigenvalues are more simply obtained as 15]

$$
\begin{aligned}
& E_{0}=E_{7}=0, \\
& E_{1}=E_{2}=E_{4}=E_{5}=-J, \\
& E_{3}=E_{6}=2 J
\end{aligned}
$$

and the corresponding eigenstates are explicitly given by

$$
\begin{aligned}
\left|\psi_{0}\right\rangle & =|000\rangle, \\
\left|\psi_{1}\right\rangle & =3^{-1 / 2}\left(q|001\rangle+q^{2}|010\rangle+|100\rangle\right), \\
\left|\psi_{2}\right\rangle & =3^{-1 / 2}\left(q^{2}|001\rangle+q|010\rangle+|100\rangle\right), \\
\left|\psi_{3}\right\rangle & =3^{-1 / 2}(|001\rangle+|010\rangle+|100\rangle), \\
\left|\psi_{4}\right\rangle & =3^{-1 / 2}\left(q|110\rangle+q^{2}|101\rangle+|011\rangle\right), \\
\left|\psi_{5}\right\rangle & =3^{-1 / 2}\left(q^{2}|110\rangle+q|101\rangle+|011\rangle\right), \\
\left|\psi_{6}\right\rangle & =3^{-1 / 2}(|110\rangle+|101\rangle+|011\rangle), \\
\left|\psi_{7}\right\rangle & =|111\rangle .
\end{aligned}
$$

with $q=\exp (i 2 \pi / 3)$ satisfying

$$
\begin{aligned}
q^{3} & =1, \\
q^{2}+q+1 & =0 .
\end{aligned}
$$

This set (4) of three-qubit states is itself interesting. Rajagopal and Rendell [10] have considered a similar set of three-qubit states which they have classified by means of permutation symmetries. Here the states $\left|\psi_{0}\right\rangle,\left|\psi_{3}\right\rangle,\left|\psi_{6}\right\rangle$, and $\left|\psi_{7}\right\rangle$ are symmetric in the permutation of any pair of particles. We define a cyclic shift operator $P$ by its action on the basis $|i j k\rangle$ [29]

$$
P|i j k\rangle=|k i j\rangle \text {. }
$$

Obviously the four states $\left|\psi_{0}\right\rangle,\left|\psi_{3}\right\rangle,\left|\psi_{6}\right\rangle$, and $\left|\psi_{7}\right\rangle$ are the eigenstates of $P$ with eigenvalue 1 . The other four states in the set (4) are also eigenstates of $P$ as follows:

$$
\begin{aligned}
& P\left|\psi_{i}\right\rangle=q^{2}\left|\psi_{i}\right\rangle(i=1,4), \\
& P\left|\psi_{j}\right\rangle=q\left|\psi_{j}\right\rangle(j=2,5) .
\end{aligned}
$$

This is not surprising since the Hamitonian $H_{X X}$ as well as the other Hamiltonians considered later are invariant under the cyclic shift operator.

For $J>0(J<0)$ the ground state is four (two)fold degenerate. We will see that the degeneracy of the system influences thermal entanglement greatly. There is no pairwise entanglement in the eigenstate $\left|\psi_{0}\right\rangle$ and $\left|\psi_{7}\right\rangle$. Pairwise entanglement exists in the state $\left|\psi_{i}\right\rangle(i=$ $1,2, \ldots, 6)$ and the concurrence between any two different qubits is given by $2 / 3$ [8,30].

\section{THERMAL ENTANGLEMENT IN THE $X X$ MODEL}

We first recall the definition of concurrence 31 between a pair of qubits. Let $\rho_{12}$ be the density matrix of the pair and it can be either pure or mixed. The concurrence corresponding to the density matrix is defined as

$$
\mathcal{C}_{12}=\max \left\{\lambda_{1}-\lambda_{2}-\lambda_{3}-\lambda_{4}, 0\right\},
$$

where the quantities $\lambda_{i}$ are the square roots of the eigenvalues of the operator

$$
\varrho_{12}=\rho_{12}\left(\sigma_{1}^{y} \otimes \sigma_{2}^{y}\right) \rho_{12}^{*}\left(\sigma_{1}^{y} \otimes \sigma_{2}^{y}\right)
$$

in descending order. The eigenvalues of $\varrho_{12}$ are real and non-negative even though $\varrho_{12}$ is not necessarily Hermitian. The values of the concurrence range from zero, for an unentangled state, to one, for a maximally entangled state.

The state at thermal equilibrium is described by the density matrix

$$
\begin{aligned}
\rho(T) & =\frac{1}{Z} \exp (-\beta H), \\
& =\frac{1}{Z} \sum_{k=0}^{7} \exp \left(-\beta E_{k}\right)\left|\psi_{k}\right\rangle\left\langle\psi_{k}\right| .
\end{aligned}
$$

where $\beta=1 / T$. From Eq.(3), the partition function is obtained as 


$$
Z=2+4 e^{\beta J}+2 e^{-2 \beta J} .
$$

¿From Eqs.(3) and (10), the density matrix can be written as

$$
\begin{aligned}
\rho(T)= & \frac{1}{Z}\left[\left|\psi_{0}\right\rangle\left\langle\psi_{0}|+| \psi_{7}\right\rangle\left\langle\psi_{7}\right|\right. \\
& +e^{\beta J}\left(\left|\psi_{1}\right\rangle\left\langle\psi_{1}|+| \psi_{4}\right\rangle\left\langle\psi_{4}\right|\right. \\
& \left.+\left|\psi_{2}\right\rangle\left\langle\psi_{2}|+| \psi_{5}\right\rangle\left\langle\psi_{5}\right|\right) \\
& \left.+e^{-2 \beta J}\left(\left|\psi_{3}\right\rangle\left\langle\psi_{3}|+| \psi_{6}\right\rangle\left\langle\psi_{6}\right|\right)\right] .
\end{aligned}
$$

In this paper we consider only pairwise thermal entanglement, and so we need to calculate the reduced density matrix $\rho_{12}(T)=\operatorname{tr}_{3}(\rho(T))$. We denote the reduced density matrix $\operatorname{tr}_{3}\left[\left|\psi_{i_{1}}\right\rangle\left\langle\psi_{i_{1}}|+\ldots+| \psi_{i_{N}}\right\rangle\left\langle\psi_{i_{N}}\right|\right]$ by $\rho_{12}^{\left(i_{1} i_{2} \ldots i_{N}\right)}$. From Eq.(A), we obtain

$$
\begin{aligned}
\rho_{12}^{(07)}= & \left(\begin{array}{llll}
1 & 0 & 0 & 0 \\
0 & 0 & 0 & 0 \\
0 & 0 & 0 & 0 \\
0 & 0 & 0 & 1
\end{array}\right), \\
\rho_{12}^{(1245)}= & \frac{2}{3}\left(\begin{array}{llll}
1 & 0 & 0 & 0 \\
0 & 2 & -1 & 0 \\
0 & -1 & 2 & 0 \\
0 & 0 & 0 & 1
\end{array}\right), \\
\rho_{12}^{(36)}= & \frac{2}{3}\left(\begin{array}{llll}
\frac{1}{2} & 0 & 0 & 0 \\
0 & 1 & 1 & 0 \\
0 & 1 & 1 & 0 \\
0 & 0 & 0 & \frac{1}{2}
\end{array}\right) \\
\rho_{12}^{(012)}= & \frac{2}{3}\left(\begin{array}{llll}
\frac{5}{2} & 0 & 0 & 0 \\
0 & 1 & -\frac{1}{2} & 0 \\
0 & -\frac{1}{2} & 1 & 0 \\
0 & 0 & 0 & 0
\end{array}\right), \\
\rho_{12}^{(12)}= & \frac{2}{3}\left(\begin{array}{llll}
1 & 0 & 0 & 0 \\
0 & 1 & -\frac{1}{2} & 0 \\
0 & -\frac{1}{2} & 1 & 0 \\
0 & 0 & 0 & 0
\end{array}\right) .
\end{aligned}
$$

The last two reduced density matrices will be used later.

¿From Eqs. (12) and (13a-13d), we obtain

$$
\begin{aligned}
\rho_{12}(T) & =\frac{1}{Z}\left(\rho_{12}^{(07)}+e^{\beta J} \rho_{12}^{(1245)}+e^{-2 \beta J} \rho_{12}^{(36)}\right) \\
& =\frac{2}{3 Z}\left(\begin{array}{cccc}
v & 0 & 0 & 0 \\
0 & w & y & 0 \\
0 & y & w & 0 \\
0 & 0 & 0 & v
\end{array}\right)
\end{aligned}
$$

with

$$
\begin{aligned}
v & =\frac{3}{2}+e^{\beta J}+\frac{1}{2} e^{-2 \beta J}, \\
w & =2 e^{\beta J}+e^{-2 \beta J} \\
y & =e^{-2 \beta J}-e^{\beta J}
\end{aligned}
$$

The square roots of the four eigenvalues of the operator $\varrho_{12}$ are

$$
\begin{aligned}
& \lambda_{1}=\frac{2(w-y)}{3 Z}, \lambda_{2}=\frac{2(w+y)}{3 Z}, \\
& \lambda_{3}=\lambda_{4}=\frac{2 v}{3 Z} .
\end{aligned}
$$

¿From Eqs.(8), (11), (14), and (16), we obtain the concurrence 23.

$$
\begin{aligned}
\mathcal{C} & =\frac{4}{3 Z} \max (|y|-v, 0) \\
& =\max \left[\frac{2\left|e^{-2 x}-e^{x}\right|-3-2 e^{x}-e^{-2 x}}{3\left(1+2 e^{x}+e^{-2 x}\right)}, 0\right]
\end{aligned}
$$

where $x \equiv \beta J=J / T$. The concurrence depends only on the ratio of $J$ and $T$. Due to symmetry under cyclic shifts, the value of the concurrence does not depend on the choice of the pair of qubits.

¿From (18) we see that entanglement appears only when

$$
\frac{2\left|z^{-2}-z\right|-3-2 z-z^{-2}}{3\left(1+2 z+z^{-2}\right)}>0
$$

or in other words

$$
2\left|z^{-2}-z\right|-3-2 z-z^{-2}>0
$$

where $z=\exp (x)$. We now consider two different cases:

Case 1. Antiferromagnetic system; $J>0 ; z^{-2}-z<0$. In this case relation (20) requires

$$
z^{-2}<-1
$$

which is impossible. So there is no entanglement when $J>0$.

Case 2. Ferromagnetic system; $J<0, z^{-2}-z>0$. Relation (20) becomes

$$
z^{-2}-4 z-3>0
$$

or

$$
f(z) \equiv 4 z^{3}+3 z^{2}-1<0 .
$$

The function $f(z)$ is an increasing function of the positive real argument $z$ and relation (23) is valid iff $0<z<z_{c}$, where the critical value $z_{c}$ determined by $f\left(z_{c}\right)=0$ is 0.4554 ; that is, $x<-0.7866$. For fixed $J$, we obtain the critical temperature $T_{c}=1.21736|J|$, above which there is no thermal entanglement. The critical temperature depends linearly on the absolute value of $J$.

In the ferromagnetic case the concurrence

$$
\mathcal{C}=\max \left[\frac{1-4 z^{3}-3 z^{2}}{3\left(1+2 z^{3}+z^{2}\right)}, 0\right]
$$

reaches its maximum value of $1 / 3$ when $z \rightarrow 0$, that is when $x \rightarrow-\infty$. Since the entanglement is a monotonic increasing function of $\mathcal{C}$ this means that the entanglement attains its maximum value for zero temperature, when 
$J$ is finite and nonzero. For finite temperatures, this maximum is also attained when $J \rightarrow-\infty$.

In summary, we find that

Theorem 1: The XX model is thermally entangled if and only if $J<-0.7866 T$; maximum entanglement is attained when $T \rightarrow 0$ or $J \rightarrow-\infty$.

The above discussion shows that in our 3-qubit model pairwise thermal entanglement occurs only in the ferromagnetic case. This result differs from that for the twoqubit $X X$ model, for which thermal entanglement exists in both the antiferromagnetic and ferromagnetic cases [15.

For the ferromagnetic case the states $\left|\psi_{3}\right\rangle$ and $\left|\psi_{6}\right\rangle$ constitute a doubly-degenerate ground state. Eq.(24) shows that the concurrence $\mathcal{C}=1 / 3$ at zero temperature. As noted in the last section the concurrence for any two qubits in the state $\left|\psi_{3}\right\rangle$ or $\left|\psi_{6}\right\rangle$ is $2 / 3$. Here the value $1 / 3$ appears due to the degeneracy. In fact, at zero temperature, the thermal entanglement can be calculated from $\rho_{12}^{(36)}(13 \mathrm{~g})$. After normalization it is easy to obtain the concurrence, which is just $1 / 3$.

\section{THE ANISOTROPIC HEISENBERG $X X Z$ MODEL}

We now consider a more general Heisenberg model, the anisotropic Heisenberg $X X Z$ model, which is described by the Hamiltonian 32 .

$$
H_{X X Z}=H_{X X}+\frac{\Delta J}{2} \sum_{n=1}^{3}\left(\sigma_{n}^{z} \sigma_{n+1}^{z}-1\right)
$$

where $\Delta$ is the anisotropy parameter. The model reduces to the $X X$ model when $\Delta=0$, and the isotropic Heisenberg $X X X$ model when $\Delta=1$.

It is straightforward to check that the added anisotropic term $H_{X X Z}-H_{X X}$ commutes with $H_{X X}$. Therefore the eigenstates of the $X X Z$ model are still given by Eq. (4), now with the different eigenvalues

$$
\begin{aligned}
& E_{0}=E_{7}=0, \\
& E_{1}=E_{2}=E_{4}=E_{5}=-2 J\left(\Delta+\frac{1}{2}\right), \\
& E_{3}=E_{6}=-2 J(\Delta-1) .
\end{aligned}
$$

Following the procedure of the previous section, we obtain the concurrence, which is of the same form as Eq.(17) with however the parameters $v, w, y$, and the partition function $Z$ now given by

$$
\begin{aligned}
v & =\frac{3}{2}+\frac{1}{2} z^{2 \Delta}\left(2 z+z^{-2}\right), \\
w & =z^{2 \Delta}\left(2 z+z^{-2}\right) \\
y & =z^{2 \Delta}\left(z^{-2}-z\right) \\
Z & =2+2 z^{2 \Delta}\left(2 z+z^{-2}\right)
\end{aligned}
$$

As in the last section, since $Z$ is always positive, we need only consider

$$
\begin{aligned}
f(\Delta, z) & \equiv|y|-v \\
& =z^{2 \Delta}\left|z^{-2}-z\right|-\frac{3}{2}-z^{2 \Delta+1}-\frac{1}{2} z^{2 \Delta-2}
\end{aligned}
$$

to determine whether entanglement occurs or not. Again, we have to consider two different cases:

Case 1. When $J>0(z>1)$, namely the antiferromagnetic $X X Z$ model, the condition on $f(\Delta, z)$ leads to

$$
z^{2 \Delta-2}=e^{2 x(\Delta-1)}<-1
$$

which is impossible. So there is no entanglement in this case, irrespective of $\Delta$.

Case 2. When $J<0(z<1)$, namely the ferromagnetic $X X Z$ model, the condition $f(\Delta, z)>0$ gives

$$
z^{2 \Delta-2}-4 z^{2 \Delta+1}-3>0 .
$$

We consider some special values of $\Delta$.

(1) $\Delta \geq 1$ : For $\Delta=1$ the relation (30) implies $z^{3}<$ $-1 / 2$ which is impossible. So there is no entanglement in the $X X X$ model. We can further prove that there is no entanglement for $\Delta>1$. In fact, it is easy to see that

$$
f(\Delta, z)<z^{2(\Delta-1)}-3<0,
$$

where we have used the inequalities $z^{2 \Delta+1}>0$ and $z^{2(\Delta-1)}<1$ for $\Delta>1$ and $z<1$. This means $\mathcal{C}=0$ and thus there is no entanglement.

(2) $\Delta=1 / 2$ : In this case 33 the entanglement condition is obtained as

$$
4 z^{3}+3 z-1<0
$$

which is an increasing function of $z$. So the model is entangled iff $0<z<z_{c} \approx 0.298$, where $z_{c}$ is determined as a root of $4 z^{3}+3 z-1=0$.

(3) $\Delta=-1 / 2$ : This is an interesting case whose importance has been emphasized recently [34]. ¿From the eigenvalues we see that the excited state of the system is 6 -fold degenerate when $\Delta=-1 / 2$. The function $f(\Delta, z)$ now reduces to $z^{-3}-7$, from which the critical values $z_{c}$ and $T_{c}$ are obtained analytically as $z_{c}=7^{-1 / 3}, T_{c}=3|J| / \ln 7 \approx 1.5417|J|$.

(4) The limit case $\Delta \rightarrow-\infty$ : The critical value $z_{c}$ is now determined by $z^{-2}-4 z=0$, i.e., $z_{c}=4^{-1 / 3}$. Therefore the critical temperature $T_{c}=3|J| / \ln 4 \approx 2.164|J|$.

Finally, for more general values of the anisotropy parameter we need to resort to numerical calculations. Fig. 1 is a plot of the critical temperature as a function of the anisotropy parameter $\Delta$. From this we see that the critical temperature decreases as $\Delta$ increases, and reaches the asymptotic value $T_{c}=2.1640|J|$ as $\Delta \rightarrow-\infty$. 
as indicated in Fig. 2.

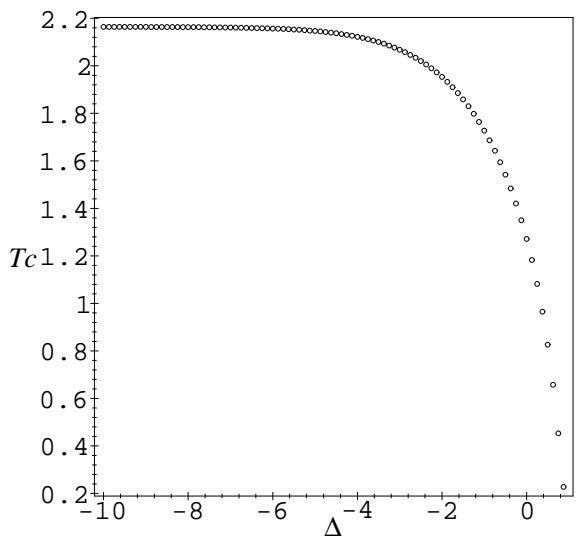

FIG. 1. The critical temperature $T_{c}$ as a function of the anisotropy parameter $\Delta$. The exchange constant $J=-1$.

We now give further analytical results for the case $\Delta<$ 1 and $z<1$. Consider $f(\Delta, z)$ as a function of $\Delta$. Then, from

$$
\begin{aligned}
\frac{\partial f(\Delta, z)}{\partial \Delta}= & (\ln z) z^{2 \Delta}\left(z^{-2}-4 z\right) \\
& \begin{cases}=0, \text { when } z=z_{0} \equiv 4^{-1 / 3} \approx 0.62996 ; \\
>0, \text { when } z>z_{0} ; \\
<0, \text { when } z<z_{0} .\end{cases}
\end{aligned}
$$

we see that $f(\Delta, z)$ is an increasing (decreasing) function when $z>z_{0}\left(z<z_{0}\right)$. We consider these cases separately.

Case 2a. When $z=z_{0}, f\left(\Delta, z_{0}\right)=-3<0$. So there is no entanglement in this case.

Case 2b. When $z>z_{0}$, the function $f(\Delta, z)$ is an increasing function which reaches its maximum when $\Delta \rightarrow 1$. Since we have seen that there is no entanglement when $\Delta=1$

$$
f(\Delta, z)<f(1, z)<0 \quad \text { for } z>z_{0},
$$

which means that there is no entanglement when $z>z_{0}$.

Case 2c. The case $z<z_{0}$. Define the $z$-dependent point $\Delta_{z}$ by $f\left(\Delta_{z}, z\right)=0$ where

$$
\Delta_{z}=\frac{1}{2 \beta J} \ln \left[\frac{3}{z^{-2}-4 z}\right]<1 .
$$

Thus from Eq.(33) we know that $f(\Delta, z)>0$ when $\Delta<\Delta_{z}$ for all $z<z_{0}$, which is just the condition for entanglement.

In Fig. 2 we give plots of $f(\Delta, z)$ for $z=$ $0.6295,0.6,0.5,0.4,0.3,0.2,0.1$. Note that $\Delta_{z}$ is a decreasing function of $z$ and that

$$
\Delta_{z} \rightarrow \begin{cases}-\infty & \text { when } z \rightarrow z_{0} \\ 1 & \text { when } z \rightarrow 0\end{cases}
$$

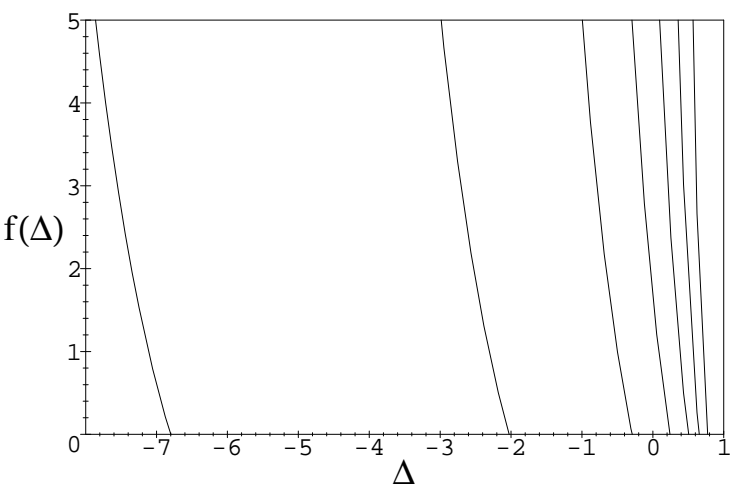

FIG. 2. The function $f(\Delta, z)$ with respect $\Delta$ for $z=0.6295,0.6,0.5,0.4,0.3,0.2,0.1$ (from left to right).

In summary, we have

Theorem 2 The $X X Z$ model exhibits thermal entanglement only when $z<z_{0}$, that is, $J<-.4621 T$, and $\Delta<\Delta_{z}$.

Note that Theorem 2 is entirely consistent with Theorem 1 , since $\Delta_{z}=0$ in Eq.(35) corresponds to $J=$ -0.78667 .

In Fig. 3 we plot the concurrence as a function of the anisotropy parameter $\Delta$ and exchange constant $J$. The figure shows that there is no thermal entanglement for the antiferromagnetic $(J>0) X X Z$ model, nor for the ferromagnetic $(J<0) X X Z$ model when $\Delta \geq 1$.

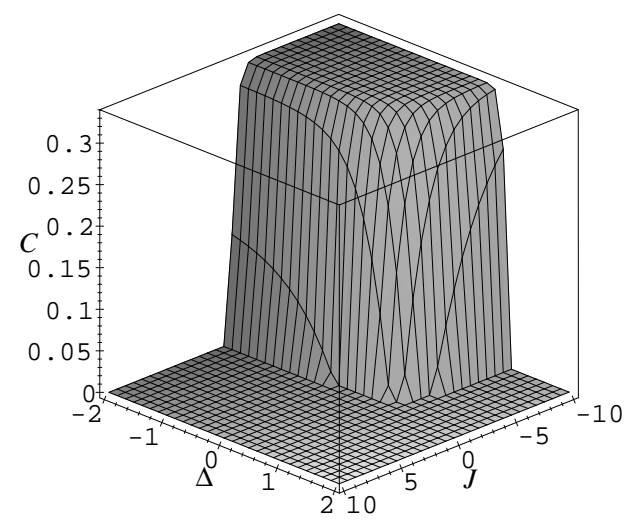

FIG. 3. The concurrence as a function of $\Delta$ and $J$. The temperature $T=1$.

To end this section we investigate the concurrence at 
zero temperature. Nonzero concurrence occurs for the case $\Delta<1, J<0$. In this case, the doubly-degenerate ground state consists of $\left|\psi_{3}\right\rangle$ and $\left|\psi_{6}\right\rangle$. We may calculate the concurrence $\mathcal{C}=1 / 3$ directly from the density matrix $\rho_{12}^{(36)}$.

\section{EFFECTS OF MAGNETIC FIELDS}

In this section we consider the effect of magnetic fields on thermal entanglement. The $X X Z$ model with uniform magnetic field $B$ along the $z$ direction is given by

$$
H_{X X Z M}=H_{X X Z}+B \sum_{n=1}^{3} \sigma_{n}^{z} .
$$

It is easy to check that the added magnetic term commutes with the Hamiltonian $H_{X X Z}$. Therefore the eigenstates of the $X X Z$ model are given by Eq.(4). The eigenvalues are now

$$
\begin{aligned}
& E_{0}=-3 B \\
& E_{1}=E_{2}=-2 J\left(\Delta+\frac{1}{2}\right)-B \\
& E_{3}=-2 J(\Delta-1)-B, \\
& E_{4}=E_{5}=-2 J\left(\Delta+\frac{1}{2}\right)+B, \\
& E_{6}=-2 J(\Delta-1)+B . \\
& E_{7}=3 B
\end{aligned}
$$

We see that the magnetic field partly removes the degeneracy.

With a derivation completely analogous to that of Sec. III and Sec. IV, the reduced density operator is

$$
\rho_{12}=\frac{2}{3 Z}\left(\begin{array}{cccc}
u & 0 & 0 & 0 \\
0 & w & y & 0 \\
0 & y & w & 0 \\
0 & 0 & 0 & v
\end{array}\right)
$$

with

$$
\begin{aligned}
u= & \frac{3}{2} e^{3 \beta B}+\frac{1}{2} e^{\beta B} z^{2 \Delta}\left(2 z+z^{-2}\right) \\
v= & \frac{3}{2} e^{-3 \beta B}+\frac{1}{2} e^{-\beta B} z^{2 \Delta}\left(2 z+z^{-2}\right) \\
w= & \cosh (\beta B) z^{2 \Delta}\left(2 z+z^{-2}\right) \\
y= & \cosh (\beta B) z^{2 \Delta}\left(z^{-2}-z\right) \\
Z= & 2 \cosh (3 \beta B)+2 \cosh (\beta B) z^{2 \Delta} \\
& \times\left(2 z+z^{-2}\right)
\end{aligned}
$$

The concurrence is then given by

$$
\mathcal{C}=\frac{4}{3 Z} \max (|y|-\sqrt{u v}, 0) .
$$

As an immediate consequence we see that the concurrence is an even function of the magnetic field.
As the quantities $Z, u, v$ are all positive, for convenience we consider the quantity $y^{2}-u v$ instead of $|y|-\sqrt{u v}$. Thermal entanglement occurs when

$$
y^{2}-u v=h \cosh (2 \beta B)-g>0,
$$

where

$$
\begin{aligned}
& g=\frac{1}{4}\left[9+z^{4(\Delta-1)}\left(2 z^{6}+8 z^{3}-1\right)\right], \\
& h=\frac{1}{2} z^{2 \Delta}\left[z^{2 \Delta}\left(z^{-2}-z\right)^{2}-\left(6 z+3 z^{-2}\right)\right] .
\end{aligned}
$$

We now consider the effect of a magnetic field on the thermal entanglement.

We first consider the $X X X$ model, $\Delta=1$, which does not exhibit thermal entanglement when $B=0$. One might expect that the magnetic field would induce thermal entanglement. It is easy to see that

$$
2\left(y^{2}-u v\right)=\cosh (2 \beta B)\left(z^{6}-8 z^{3}-2\right)-\left(z^{3}+2\right)^{2} .
$$

If $z<(4+3 \sqrt{2})^{1 / 3} \approx 2.02, z^{6}-8 z^{3}-2<0$ and thus $y^{2}-u v<0$ for any $B$. So for this range of $z$ values there is no thermal entanglement no matter how strong the magnetic field is. However, when $z>(4+3 \sqrt{2})^{1 / 3}$, $z^{6}-8 z^{3}-2>0$ and the condition for entanglement becomes

$$
\cosh (2 \beta B)>\frac{\left(z^{3}+2\right)^{2}}{z^{6}-8 z^{3}-2}
$$

which can be fulfilled for strong enough $B$. So a magnetic field can induce entanglement in the $X X X$ model when $z>(4+3 \sqrt{2})^{1 / 3}$.

Now consider the case $\Delta=-1 / 2$. ¿From Eq. (43) we obtain

$$
\begin{aligned}
h & =\frac{1}{2}\left(p^{2}-5 p-5\right), \\
g & =\frac{1}{4}\left(11+8 p-p^{2}\right), \\
h-g & =\frac{1}{4}\left(3 p^{2}-18 p-21\right),
\end{aligned}
$$

which are parabolas in $p \equiv z^{-3}$, as shown in Fig. 4 . We consider three different cases:

Case 1: $p<p_{1}=5 / 2+3 \sqrt{5} / 2$, In this case $h<$ $0, g>0, h-g<0$ and $y^{2}-u v<0$. So there is no thermal entanglement.

Case 2: $p_{1}<p<p_{2}=7$. In this case $h>0, g>$ $0, h-g<0$. So $y^{2}-u v>0$, and so entanglement appears if the magnetic field is strong enough.

Case 3: $p_{2}<p$. In this case $h>0, h-g>0$ and $y^{2}-u v$ is always positive; that is, here the XXZ model exhibits thermal entanglement for any magnetic field. Note that $p_{2}=z_{c}^{-3}$ where $z_{c}$ is the critical value given in last section. 
The above two models show that the magnetic field can either induce entanglement in a non-entangled system or extend the entanglement range for an already entangled system.

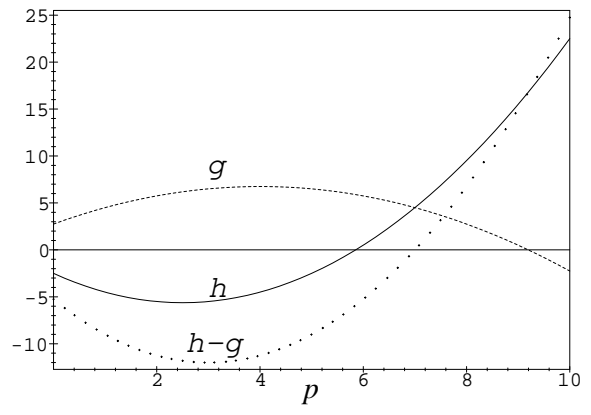

FIG. 4. The functions $h, g$, and $h-g$ in terms of $p=z^{-3}$.

In Fig. 5 we plot the concurrence as a function of the magnetic field $B$ and exchange constant $J$. At $B=0$ there is no thermal entanglement. The entanglement increases with the magnetic field $|B|$ until it reaches a maximum value, then decreases and gradually disappears. We can clearly see that there is no thermal entanglement for the ferromagnetic case, while thermal entanglement exists for the antiferromagnetic case. In other words, we can induce entanglement in the antiferromagnetic $X X X$ system by introducing a magnetic field, but cannot induce entanglement in the ferromagnetic $X X X$ system for any strength of magnetic field.

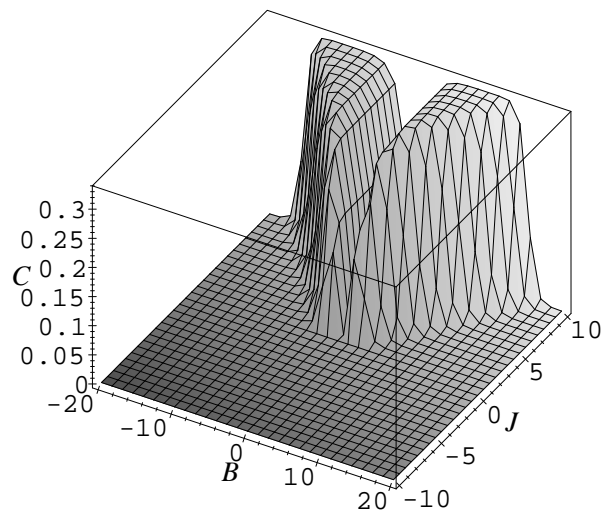

FIG. 5. Concurrence as a function of the magnetic field $B$ and the exchange constant $J$. The temperature $T=1$ and the anisotropy parameter $\Delta=1$.

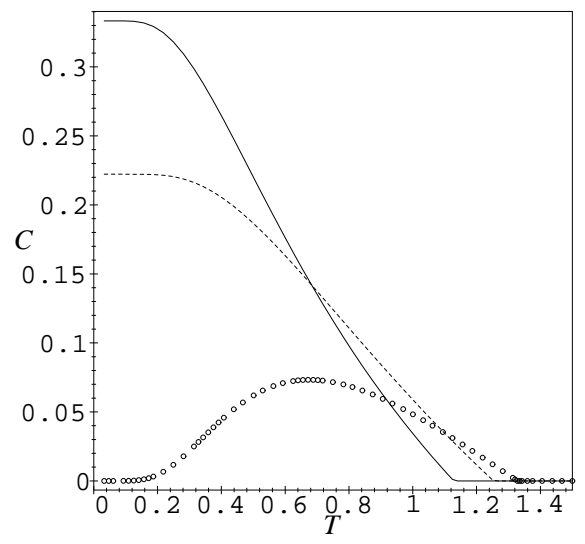

FIG. 6. Concurrence as a function of $T$ for different magnetic fields $B=1$ (solid line), $3 / 2$ (dashed line), and 2 (circle point line).

Fig. 6 gives a plot of the concurrence as a function of the temperature for different magnetic fields. One can see that there exist critical temperatures above which the entanglement vanishes. It is also noteworthy that the critical temperature increases as the magnetic field $B$ increases. Consider the interesting case $B=2$. We observe that the concurrence is zero at zero temperature and there is a maximum value of concurrence at a finite temperature. The entanglement can be increased by increasing the temperature. The maximum value is due to the optimal mixing of all eigenstates in the system. When considering zero temperature we find that there are different limits for different magnetic fields. Actually a more general result exists

$$
\begin{aligned}
\lim _{T \rightarrow 0} \mathcal{C}(\Delta, B, 1, T) & =\frac{1}{3} \text { for } \Delta>|B|-1 / 2 . \\
& =\frac{2}{9} \text { for } \Delta=|B|-1 / 2 \\
& =0 \text { for } \Delta<|B|-1 / 2 .
\end{aligned}
$$

The special point $T=0, \quad \Delta=B-1 / 2(B \geq 0$ is assumed without loss of generality), at which the entanglement undergoes a sudden change with adjustment of the parameters $\Delta$ and $B$, is the point of quantum phase transition [35]. The quantum phase transition takes place at zero temperature due to the variation of interaction terms in the Hamiltonian. By examining the eigenvalues (38) we can understand the phase transition. When $\Delta=B-1 / 2$, the ground state contains 
the three-fold degenerate states $\left|\psi_{0}\right\rangle,\left|\psi_{1}\right\rangle$, and $\left|\psi_{2}\right\rangle$. One may calculate the thermal entanglement from the density matrix $\rho_{12}^{(012)}(13 \mathrm{~d})$ and find the concurrence to be $2 / 9$. When $\Delta>B-1 / 2$, the ground state contains the two-fold degenerate states $\left|\psi_{1}\right\rangle$ and $\left|\psi_{2}\right\rangle$. The concurrence has the value $1 / 3$ as calculated from $\rho_{12}^{(12)}$ (13e). When $\Delta<B-1 / 2$, the ground state is $\left|\psi_{0}\right\rangle$ and not degenerate. And the concurrence is zero in this case.

\section{CONCLUSIONS}

Apart from being a fundamental property of quantum mechanics, it appears that entanglement may provide an important resource in quantum information processes. One source of entanglement is provided by magnetic systems, such as those modelled in this paper. Within the current state of knowledge, only measures for pairwise entanglement are available. Thus, in order to study the entanglement properties of systems more complex than those simply involving two qubits, it is necessary to adopt a procedure whereby one traces out a subsystem, leaving effectively only a two-qubit system for which we can calculate the concurrence, which in turn gives a measure of the entanglement. Using this procedure, we have studied pairwise thermal entanglement in the following Heisenberg models; the $X X$ model, the $X X Z$ model and the $X X Z$ model in a magnetic field. We obtained analytical expressions for the concurrence, which indicated no thermal entanglement for the antiferromagnetic $X X Z$ model, nor for the ferromagnetic $X X Z$ model when the anisotropy parameter $\Delta \geq 1$. Conditions for the existence of thermal entanglement were studied in detail. The effects of magnetic fields on entanglement were also considered. We found that the magnetic field can induce entanglement in the antiferromagnetic $X X X$ model, but cannot induce entanglement in the ferromagnetic $X X X$ model, no matter how strong the magnetic field is.

In this paper we have extended previous work on thermal entanglement from two-qubit models to three qubit models, concentrating on those systems where the pairwise entanglement can be studied analytically. It would be an attractive proposition to extend further the investigation of such Heisenberg models to the $N$-qubit case, which are under consideration.

\section{ACKNOWLEDGMENTS}

X. Wang thanks K. Mølmer, A. Sørensen, W. K. Wootters and Paolo Zanardi for many valuable discussions. He is supported by the Information Society Technologies Programme IST-1999-11053, EQUIP, action line 62-1 and European project Q-ACTA. H. Fu is supported in part by the National Natural Science Foundation of China (19875008), and A. I. Solomon acknowledges the hospitality of the Laboratoire de Physique Théorique des Liquides, Paris University VI.

[1] C. H. Bennett and D. P. DiVincenzo, Nature 404, 247 (2000).

[2] P. Zanardi, C. Zalka, and L. Faoro, Phys. Rev. A 62, 030301(R) (2000); P. Zanardi, Phys. Rev. A 63, 040304(R) (2001).

[3] W. Dür , G. Vidal, J. I. Cirac, N. Linden and S. Popescu, quant-ph/0006034.

[4] J. I. Cirac, W. Dür, B. Kraus and M. Lewenstein, Phys. Rev. Lett. 86, 544 (2001).

[5] J. Schliemann, J. I. Cirac, M. Kuś, M. Lewenstein, and D. Loss, Phys. Rev. A 64, 022303 (2001).

[6] Y. S. Li, B. Zeng, X. S. Liu, and G. L. Long, Phys. Rev. A 64, 054302 (2001).

[7] P. Zanardi, quant-ph/0104114.

[8] W. Dür, G. Vidal, and J. I. Cirac, Phys. Rev. A 62, 062314 (2000).

[9] V. Coffman,J. Kundu, W. K. Wootters, Phys. Rev. A 61,052306 (2000); A. Sudbery, J. Phys. A: Math. Gen. 34, 643 (2001); H. A. Carteret and A. Sudbery, quantph/0001091; T. A. Brun and O. Cohen, Phys. Lett. A 281, 88 (2001); A. Acín, A. Andrianov, L. Costa, E. Jané, J. I. Latorre, and R. Tarrach, Phys. Rev. Lett. 85, 1560 (2000); A. Acín, A. Andrianov, E. Jané, and R. Tarrach, quant-ph/0009107.

[10] A. K. Rajagopal and R. W. Rendell, quant-ph/0104122.

[11] A. Karlsson and M. Bourennane, Phys. Rev. A 58, 4394 (1998); V. N. Gorbachev and A. I. Trubilko, JETP 91, 894 (2000).

[12] J. C. Hao, C. F. Li, and G. C. Guo, Phys. Rev. A 63, 054301 (2001).

[13] D. Bruß,D. P. DiVincenzo, A. Ekert, C. A. Fuchs, C. Macchiavello, and J. A. Smolin, Phys. Rev. A 57, 2368 (1998).

[14] M. A. Nielsen, Ph. D thesis, University of Mexico, 1998, quant-ph/0011036; M. C. Arnesen, S. Bose, and V. Vedral, quant-ph/0009060.

[15] X. Wang, Phys. Rev. A 64, 012313 (2001).

[16] X. Wang, Phys. Lett. A 281, 101 (2001).

[17] D. Gunlycke, S. Bose, V. M. Kendon, and V. Vedral, Phys. Rev. A 64, 042302 (2001).

[18] D. Loss and D. P. Divincenzo, Phys. Rev. A 57, 120 (1998); G. Burkard, D. Loss and D. P. DiVincenzo,Phys. Rev. B 59, 2070 (1999).

[19] B. E. Kane, Nature 393, 133 (1998).

[20] R. Vrijen et al., quant-ph/9905096.

[21] Anders Sørensen and Klaus Mølmer, Phys. Rev. Lett. 83, 2274 (1999).

[22] D. A. Lidar, D. Bacon, and K. B. Whaley, Phys. Rev. Lett. 82, 4556 (1999); D. P. DiVincenzo, D. Bacon, J. Kempe, G. Burkard, and K. B. Whaley, Nature 408, 339 (2000)

[23] K. M. O'Connor, W. K. Wootters, Phys. Rev. A 63, 
$052302(2001)$.

[24] E. Lieb, T. Schultz, and D. Mattis, Ann. Phys. (N. Y.) 16, 407 (1961).

25] V. Privman, I. D. Vagner and G. Kventsel, quant$\mathrm{ph} / 9707017$.

[26] S. B. Zheng, G. C. Guo, Phys. Rev. Lett. 85, 2392 (2000).

[27] A. Imamoḡlu, D. D. Awschalom, G. Burkard, D. P. DiVincenzo, D. Loss, M. Sherwin, and A. Small, Phys. Rev. Lett. 83, 4204 (1999).

[28] P. Jordan and E. Wigner, Z. Phys. 47, 631 (1928).

[29] J. Schnack, cond-mat/0006317.

[30] M. Koashi, V. Bužek, and N. Imoto, Phys. Rev. A 62, 050302 (2000).

[31] S. Hill and W. K. Wootters, Phys. Rev. Lett. 78, 5022 (1997); W. K. Wootters, Phys. Rev. Lett. 80, 2245; V. Coffman, J. Kundu, and W. K. Wootters, Phys. Rev. A 61, 052306 (2000).

[32] R. Orbach, Phys. Rev. 112, 309 (1958);L. R. Walker, Phys. Rev. 116, 1089 (1959); J. des Cloizeaux and M. Gaudin, J. Math. Phys. 7, 1384 (1966); C. N. Yang and C. P. Yang, Phys. Rev. 150, 321 (1966)

[33] V. Fridkin, Yu. Stroganov and D. Zagier, J. Phys. A: Math. Gen. 33, L121 (2000).

[34] Yu.G. Stroganov, J.Phys. A: Math.Gen. 34, L179 (2001); A. V. Razumov and Yu. G. Stroganov, J. Phys. A: Math. Gen. 343185 (2001); M. T. Batchelor, J. de. Gier, and B. Nienhuis, cond-mat/0101385.

[35] S. Sachdev, Quantum phase transitions (Cambridge University Press, Cambridge, 1999). 